\title{
Uniform subdivision algorithms for curves and surfaces
}

N. Dyn, J.A. Gregory* and D. Levin 
N. DYN ${ }^{\dagger}$, J.A. GREGORY* and D. LEVIN ${ }^{\dagger}$

${ }^{\dagger}$ School of Mathematical Sciences

Tel-Aviv University

* Department of Mathematics and Statistics

Brunel University

Abstract A convergence analysis for studying the continuity and differentiability of limit curves generated by uniform subdivision algorithms is presented. The analysis is based on the study of corresponding difference and divided difference algorithms. The alternative process of "integrating" the algorithms is considered. A specific example of a 4-point interpolatory curve algorithm is described and its generalization to a surface algorithm defined over a subdivision of a regular triangular partition is illustrated. Key words: Subdivision algorithms, Control polygon, Interpolation, Shape control.

\section{Introduction}

Subdivision algorithms which generate curves and surfaces play an important role in the subject of computer aided geometric design. The basic idea is that a given initial "control polygon" is successively refined so that, in the limit, it approaches a smooth curve or surface. We will consider uniform binary subdivision algorithms for curves of the following form:

At the $k+1$ 'st step of the algorithm, $k=0,1,2, \ldots$, let $\mathrm{f}^{\mathrm{k}}$ denote 
the control polygon in $R^{N}$ with "control point" vertices $f \frac{k}{i} \in R^{N}, i \in Z$

Then the control polygon $\mathrm{f}^{\mathrm{k}+1}$ has vertices defined by the rule

$$
\left.\begin{array}{l}
f_{2 i}^{k+1}=\sum_{j=\ell}^{m} a_{j} f_{i}^{k}+j^{\prime} \\
f_{2 i+1}^{k+1}=\sum_{j=\ell}^{m} b_{j} f_{i+j}^{k}
\end{array}\right\} i \in z,
$$

Where $\ell<\mathrm{m}$

Our motivation for studying uniform subdivision schemes of the form (1) is based on the particular example of a 4-point interpolatory rule defined by

$$
\left.\begin{array}{l}
f_{2 i}^{k+1}=f_{i}^{k}, \\
f_{2 i+1}^{k+1}=\left[\frac{1}{2}+w\right]\left[f \frac{k}{i}+f_{i+1}^{k}-w\left[f_{i-1}^{k}+f_{i+2}^{k}\right.\right.
\end{array}\right\}
$$

see Dyn, Gregory, Levin (1987). Here, w acts as a shape control parameter. The case $\mathrm{w}=0$, namely

$$
\left.\begin{array}{l}
\mathrm{f} \frac{\mathrm{k}+1}{2 i}=\mathrm{f}_{i}^{\mathrm{k}} \\
\mathrm{f}_{2 \mathrm{i}+1}^{\mathrm{k}+1}=\frac{1}{2}\left[\mathrm{f}_{i}^{\mathrm{k}}+\mathrm{f}_{i+1}^{\mathrm{k}}\right]
\end{array}\right\}
$$

has control polygons $\mathrm{f}^{\mathrm{k}+1}=\mathrm{f}^{\mathrm{k}}$ for all $\mathrm{k}$, hence in this case the limit curve is the initial control polygon $f^{0}$. The need to construct a convergence theory for the more general case $\omega \neq 0$ leads us to consider the more general form (1).

Figure 1 illustrates the application of the interpolatory subdivision scheme defined by (2) to a finite open polygon in $\mathrm{R}^{2}$, where $\omega=1 / 16$. (The case $\omega=1 / 16$ is of significance, since it gives a rule which reproduces cubic polynomials with respect to data defined on a diadic point parameterization.) It should be noted that, since the 
binary subdivision scheme is local, the scheme is well defined in the case of finite initial data, where control points at each end of the initial polygon act as end conditions on the final limit curve.
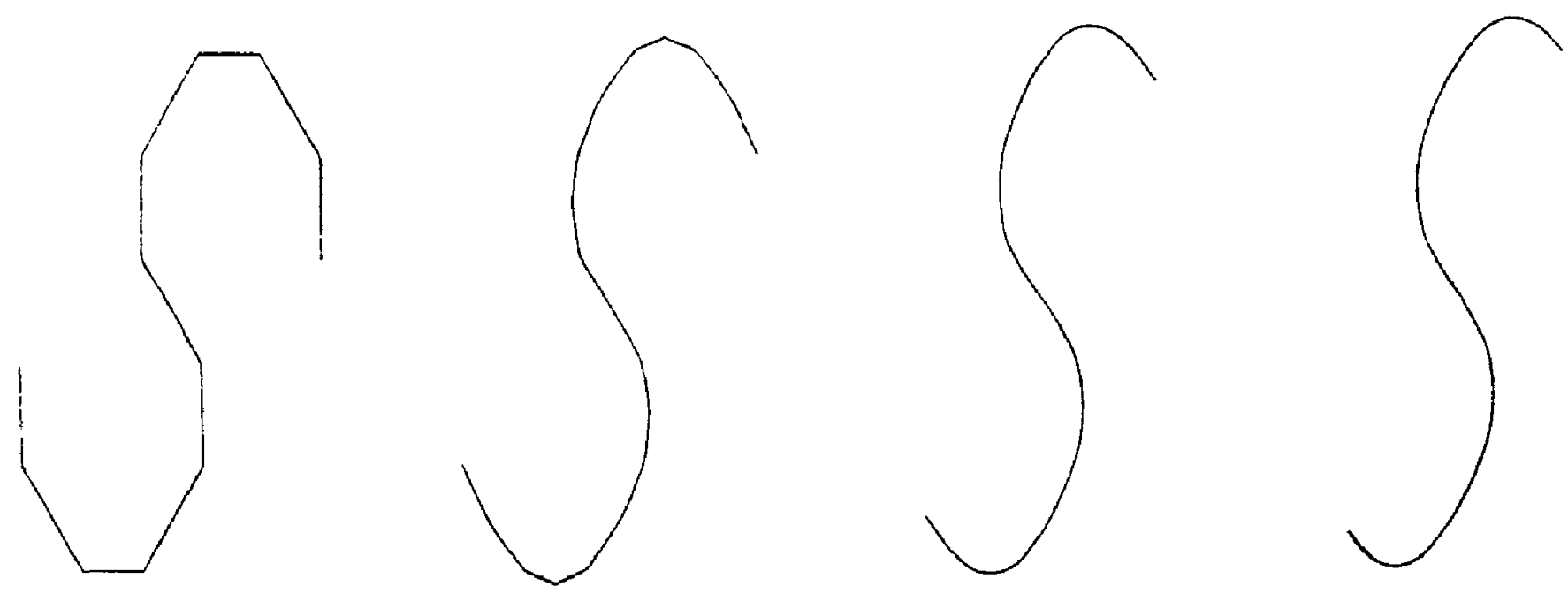

Figure 1. Example of 4-point interpolatory algorithm

Subdivision algorithms have been studied in a general setting by Micchelli and Prautzsch (1987a, 1987b, 1987c). Here, however, we review a convergence analysis presented in Dyn, Gregory and Levin (1988), for schemes defined by a rule of the specific form (1). The reader is referred to the 1988 paper for many of the proofs and this allows us to simplify the presentation. Also, our approach here is different in that the analysis is presented as a study of a fundamental solution of the scheme. In the final section a new interpolatory subdivision scheme for surfaces is introduced.

For the purposes of the analysis we will consider, without loss of generality, the basic scheme (1) with $\ell=0$. This scheme with coefficients $a=\left[a_{0} \ldots, a_{m}\right]$ and $b=[b o \ldots, b m]$ will be denoted

symbolically by $S(a, b)$. Thus $S(a, b)$ with initial data $\left\{f_{i}^{0} \in R^{N} /\right.$

$i \in$ Z $\}$ is defined by the rule 


$$
\left.\begin{array}{l}
f_{2 i}^{k+1}=\sum_{j=0}^{m} a_{j} f_{i+1}^{k} \\
f_{2 I+1}^{k+1}=\sum_{J=0}^{M} b_{j} f_{i+j}^{k}
\end{array}\right\} \quad i \in z, k=0,1,2, \ldots
$$

\section{The fundamental solution}

In order to study the limiting behaviour of the sequence of control polygons $\left\{\mathrm{f}^{\mathrm{k}}\right\}_{\mathrm{K}=0}^{\infty}$ produced by the scheme $\mathrm{S}(\mathrm{a}, \mathrm{b})$ applied to the initial Data $\left\{f_{i}^{0} / i \in Z\right\}$ we will consider the special case of initial data $\left\{\delta_{i, 0} / i \in Z\right\}$ We first, however, describe a parameterization by which the control polygons can be represented in function form. Since the process is one of binary subdivision, the initial controll points $f_{i}^{0}$ are associated wthi the integer mesh points $t_{i}^{0}:=i$, $i \in Z$, and in general, the control points $f_{i}^{k}$ are associated with the diadic mesh points

$$
t_{i}^{k}=i / 2^{k}, \quad i \in z, k=0,1,2, \ldots .
$$

The polygon $\mathrm{f}^{\mathrm{k}}$ can now be represented by the piecewise linear Inter-polant

$$
f^{k}(t):=\left(\begin{array}{c}
t^{k} i+1-t \\
t_{i+1}^{k}-t_{i}^{k}
\end{array}\right) f_{i}^{k}+\left(\frac{t-t_{i}^{k}}{t^{k}} \frac{f_{i+1}^{k}+t \frac{k}{i}}{i+1}\right) t\left(t_{i}^{k}, t_{i+1}^{k}\right), i \quad \in z .
$$

and properties of the limit process can be studied with respect to the parameterization $t$.

Let $\varphi^{k}(t)$ be the control polygon with vertices $\left\{\varphi_{i}^{k} / i \in z\right\}$ for the process $S(a, b)$ applied to the initial data $\left\{\varphi_{i}^{\circ}=\delta_{i, o} / i \in z\right\}$. Thus 


$$
\left.\begin{array}{l}
\varphi_{2 i}^{k+1}=\sum_{j=0}^{m} a_{j} \varphi_{i+j}^{k} \\
\varphi_{2 i+1}^{k+1}=\sum_{j=0}^{m} b_{j} \varphi_{i+j}^{k}
\end{array}\right\}, \quad i \in z, k=0,1,2, \ldots,
$$

where

$$
\varphi_{i}^{0}=\left\{\begin{array}{l}
1 \text { for } i=0 \\
\text { 0otherwise }
\end{array}\right.
$$

Then

$$
f^{k}(t)=\sum_{i \in z} f_{i}^{0} \varphi^{k}(t-i)
$$

is the control polygon with vertices $\left\{f_{i}^{k} / i \in z\right\}$ for arbitrary initial $\operatorname{data}\left\{f_{i}^{0} / i \in z\right\}$ Suppose

$$
\lim _{k \rightarrow \infty} \varphi^{k}(t)=\varphi(t)
$$

uniformly on $\mathrm{C}(-\infty, \infty)$. Then we $\operatorname{call} \varphi(t)$ the fundamental solution of the binary subdivision process $S(a, b)$ and $\varphi^{k}(t)$ the $k^{\prime}$ th discrete fundamental solution of the process.

The local nature of the subdivision process is reflected in the fact that $\varphi^{k}$ and $\varphi$ have local support. The support of $\varphi^{k}$ is contained in the support of $\varphi$ which is at most

$$
\left.\begin{array}{l}
(-2 m, 1) \quad \text { for } a_{m} \neq 0 \\
(-2 m+1), 1) \text { for } a_{m}=0
\end{array}\right\}
$$

(In calculating the support we assume $b_{m} \neq 0$ without loss of generality since otherwise the equivalent process $\mathrm{S}(\mathrm{b}, \mathrm{a})$ can be considered.) It follows that if $\lim \varphi^{k}=\varphi$, then 


$$
\lim f^{k}(t)=\sum_{i \in z} f_{i}^{0} \varphi(t-1)
$$

Hence the convergence properties of the algorithm for arbitrary initial data are determined by the nature of the convergence of the sequence $\left\{\varphi^{\mathrm{k}}\right\}^{\infty} \mathrm{k}=0$ to $\left.\varphi\right\}$

\section{Conditions for a $\mathrm{c}^{\ominus}$ limit}

We consider conditions under which the sequence of control polygons $\left\{\varphi^{\mathrm{k}}\right\}^{\infty} \mathrm{k}=0$ converges uniformly to a continuous limit curve $\varphi$. From (7) we immediately obtain:

Lemma 1. A. necessary condition for uniform convergence to a continuous limit curve with respect to the diadic point parameterization is that

$$
\sum_{j=0}^{m} a_{j}=\sum_{j=0}^{m} b_{j}=1
$$

Assuming the necessary condition (13), we then obtain from (7) the

\section{difference scheme}

$$
\left.\begin{array}{l}
\Delta \varphi_{2 i}^{\mathrm{k}+1}=\sum_{j=0}^{\mathrm{m}} \mathrm{c}_{j} \Delta \varphi_{i+j}^{\mathrm{k}} \\
\Delta \varphi_{2 i+1}^{\mathrm{k}+1}=\sum_{j=0}^{m} \mathrm{~d}_{j} \Delta \varphi_{i+j}^{\mathrm{k}}
\end{array}\right\}, i \in \mathrm{z}, \mathrm{k}=0,1,2, \ldots
$$

where

$$
c_{j}=\sum_{i=0}^{j}\left(a_{i}-b_{i}\right), d_{j} \sum_{i=0}^{j}\left(b_{i}-a_{i}\right)+a_{j} \cdot
$$

Here 


$$
\Delta \varphi_{i}^{\mathrm{k}}:=\varphi_{i+1}^{\mathrm{k}}-\varphi_{i}^{\mathrm{k}}
$$

defines a forward difference and we denote the scheme symbolically by $\Delta S(\mathrm{a}, \mathrm{b}):=\mathrm{S}(\mathrm{c}, \mathrm{d})$. We now have the following convergence theorem:

Theorem 2 (convergence) The process $S(a, b)$ has a discrete fundamental solution sequence $\{\varphi \mathrm{k}\}^{\infty} \mathrm{k}=0$ which converges uniformly to a con-tinuous fundamental solution $\varphi$, if and only if the difference process $\Delta S(a, b)$ has a discrete fundamental sequence $\{\varphi \mathrm{k}\}^{\infty} \mathrm{k}=0$ say, which converges uniformly to the zero function $\theta(t)=0$. Proof. It is sufficient to consider the function sequences defined on the largest possible domain of local support [-2m,1]. Observe

also that the difference process (14) has control polygons $\varphi^{k}\left(t+1 / 2^{k}\right)-\varphi^{k}(t)=\theta^{k}(t+1)-\theta^{k}(t), \quad k=0,1,2 \ldots$.

Suppose $\lim \varphi^{\mathrm{k}}=\varphi$ uniformlyin $\mathrm{C}[-2 \mathrm{~m}, 1]$. Then, from (17), $\theta^{k}(t+l)-\theta^{k}(t)$ converges uniformly to zero. Now

$\theta^{k}(t)=\theta^{k}(t)-\theta^{k}(t+2 m+i)=\sum_{i=0}^{2 m}\left\{\theta^{k}(t+i)-\theta^{k}(t+i+i)\right\}, \quad t \in[-2 m, 1]$

since $\theta^{k}(t+2 m+1)$ has local support within $(1,2 m+2)$. Hence $\theta^{k}(t)$ converges uniformly to zero. Conversely, suppose lim $\theta^{k}=\theta$, $\theta(t)=0$, uniformly in $\mathrm{C}[-2 \mathrm{~m}, 1]$. Then the control polygons (17) of the difference process (14) converge uniformly to zero. Consider this difference process from level $k$ to $k+L$. Then the control polygon at level $\mathrm{k}+\mathrm{L}$ can be represented as

$$
\varphi^{\mathrm{k}+\mathrm{L}}\left(\mathrm{t}+1 / 2^{\mathrm{k}+\mathrm{L}}\right)-\varphi^{\mathrm{k}+\mathrm{L}}(\mathrm{t})=\sum_{i \in \mathrm{z}} \Delta \varphi_{i}^{\mathrm{k}} \theta^{\mathrm{L}}\left(\mathrm{t} 2^{\mathrm{k}}-i\right)
$$

cf. (9)). In particular, 


$$
\begin{gathered}
\Delta \varphi_{j}^{\mathrm{k}+\mathrm{L}}=\sum_{i \in z} \Delta \varphi_{i}^{\mathrm{k}} \theta^{\mathrm{L}}\left(\mathrm{t}_{j}^{\mathrm{k}+\mathrm{L}} 2 \mathrm{k}-\mathrm{i}\right), \\
\quad=\sum_{i=j_{0}}^{j_{0}+2 \mathrm{~m}} \Delta \varphi_{i}^{\mathrm{k}} \theta^{\mathrm{L}}\left(j / 2^{\mathrm{L}}-i\right)
\end{gathered}
$$

where $j_{0}=\left[\mathrm{J} / 2^{\mathrm{L}}\right]$ and the summation is restricted to a finite set of $2 m+1$ integers since $\theta^{\mathrm{L}}$ has local support within $(-2 \mathrm{~m}, 1)$. Since $\theta^{\mathrm{L}}$ converges uniformly to zero it follows that given $0<\alpha<1$, there exists L such that

$$
\max _{j}\left|\Delta \varphi_{j}^{k+L}\right|<\underset{j}{\max } \mid \Delta \varphi_{j}^{k}
$$

It can now be shown that this contractive property of the differences implies that $\left\{\varphi^{\mathrm{k}}\right\}_{\mathrm{k}=0}^{\infty}$ defines a Cauchy sequence in $\mathrm{C}[-2 \mathrm{~m}, 1]$ (see Dyn, Gregory, Levin (1988)) which completes the proof.

4. A matrix analysis of convergence

Observe from (15) that $c_{m}=0$ and $d_{m}=a_{m}$ in the difference scheme (14) and define the $\mathrm{n}_{1+1}$ difference vector

$$
\Delta i, k:=\left[\Delta f_{i}^{k}, \ldots, \Delta f_{i+n 1}^{k}\right] T
$$

where

$$
\mathrm{n} 1=\left\{\begin{array}{l}
2 m-1 \text { if } a_{m} \neq 0 \\
2 m-2 i f a_{m}=0 .
\end{array}\right.
$$

Then the difference scheme (14) gives the two "even" and "odd" matrix transformations

$$
\Delta_{2 i, k+1}=C_{0} \Delta_{i, k}, \Delta_{2 i+1, k+1}=C_{1} \Delta_{i, k^{\prime}}
$$

Where 


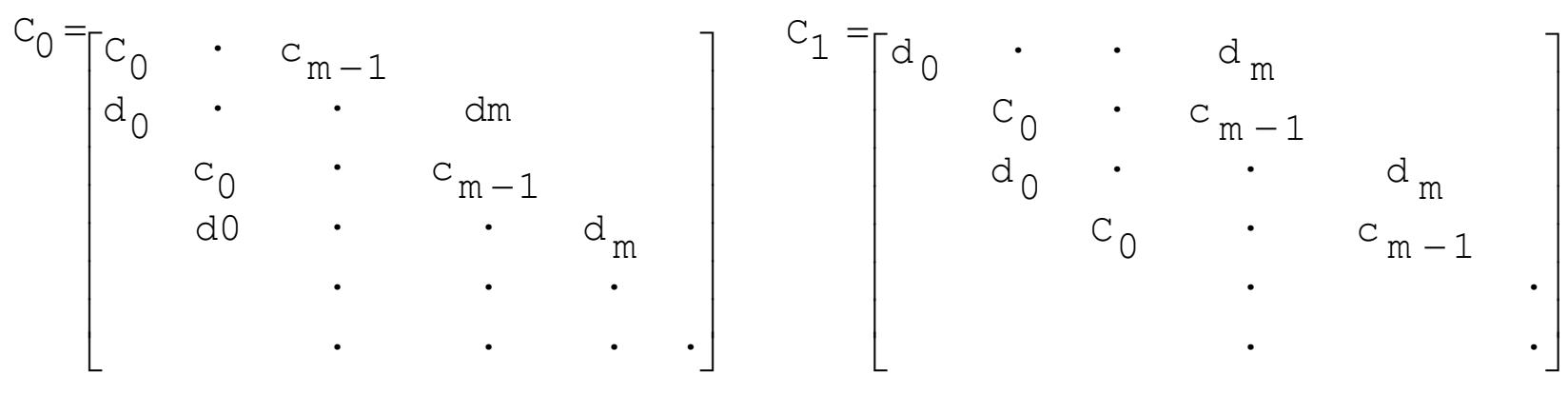

We call the $\left(\mathrm{n}_{1}+1\right) \times\left(\mathrm{n}_{1}+1\right)$ matrices $\mathrm{C}_{0}$ and $\mathrm{C}_{1}$ the control point matrices of the difference scheme $\Delta \mathrm{S}(\mathrm{a}, \mathrm{b})$. (Here, $\mathrm{n}_{1}$ has been calculated to give square matrices of lowest possible order.)

From (21) it follows that all transformations between the k'th and k+L'th differences can be accomplished by transformation matrices consisting of all permutations of products of length $L$ of the matrices $\mathrm{C}_{0}$ and $\mathrm{C}_{1}$. The contractive property (18) in the proof of Theorem 2 then leads to the following:

Theorem 3 (convergence) The discrete fundamental solutions of $\Delta \mathrm{S}(\mathrm{a}, \mathrm{b})$ converge uniformly to zero if and only if given $0<\alpha<1$, there exists a positive integer $\mathrm{L}$ such that

$$
\left\|C_{j_{L}} \ldots C_{i_{1}}\right\|_{\infty} \leq \alpha \quad \forall i_{j} \in\{0,1\}, j=1, \ldots, L .
$$

Corollary 4 A necessary condition that the discrete fundamental solutions converge uniformly to zero is that the spectral radii of $\mathrm{C}_{0}$

and $C_{1}$ satisfy

$$
\mathrm{p}\left(\mathrm{C}_{0}\right)<1 \text { and } \mathrm{p}\left(\mathrm{C}_{1}\right)<1 .
$$

The analysis is, in fact, very rich in matrix theory. For example $\mathrm{C}_{0}$ and $\mathrm{C}_{1}$ share nearly all common eigenvalues. They also share common eigenvalues with the $\left(n_{1}+2\right)$ x $\left(n_{1}+2\right)$ control matrices, $A_{0}$ and $A_{1}$ say, of the basic scheme $S(a, b)$ (excluding the one eigenvalue unity given by the necessary condition (13)). For details of these results we refer the reader to Dyn et al (1988).

( See also 
Micchelli and Prautzsch (1987a) for their treatment in terms of invariant subspaces.)

The difference scheme $\Delta S(a, b)$ has control point matrices of one less order than the basic scheme $S(a, b)$. Likewise, if there exist higher order difference schemes these will have control point matrices of lower order. This suggests the application of Theorem 3 to such higher order difference schemes since we have:

Theorem 5 Assume the necessary condition (24) and that there exists the difference scheme $\Delta l \mathrm{~S}(\mathrm{a}, \mathrm{b}), \mathfrak{l} \geq 1$. Then the difference process $\Delta S(a, b)$ has discrete fundamental solutions which converge uniformly to zero if and only if $\Delta l \mathrm{~S}(\mathrm{a}, \mathrm{b})$ has discrete fundamental solutions which converge uniformly to zero.

Remarks 6. Given $\Delta \ell S(a, b)$, the existence of $\Delta \ell S(a, b)$ requires that the sum of the coefficient vectors of $\Delta^{\ell-1} \mathrm{~S}(\mathrm{a}, \mathrm{b})$ be identical.For example, if $\Sigma c j=\Sigma d j$ for the difference scheme $A S(a, b)=S(c, d)$, then there exists the scheme $\Delta^{2} s(a, b)=\Delta S(c, d)$. Moreover, these sums will be eigenvalues of the control point matrices $\mathrm{C}_{0}$ and $\mathrm{C}_{1}$ and must thus have magnitude less than unity by the necessary condition (24) .

5. Conditions for a $C^{\ell}$ limit

To study differentiability of the limit process we consider the behaviour of the divided differences

$$
a_{i}^{k}:=\left[\varphi_{i+1}^{k}-\varphi_{i}^{k}\right] /\left[t_{i+1}^{k}-t_{i}^{k}\right]=2^{k} \Delta \varphi_{i}^{k}
$$

of the vertices of the control polygon $\varphi^{k}$. From (14) it follows that the divided differences satisfy the scheme 


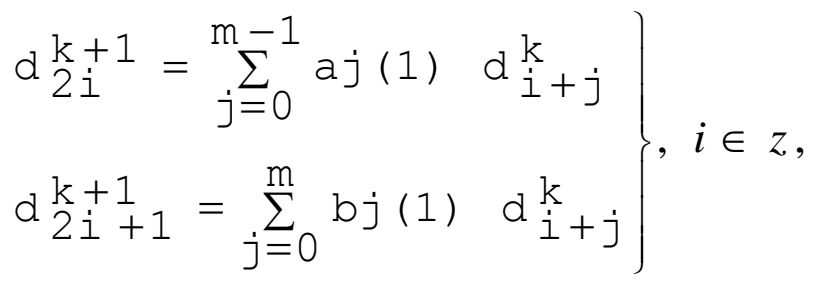

where

$a_{j}^{(1)}=2 c_{j}=2 \sum_{i=0}^{j}\left(a_{i}-b_{i}\right), b_{j}^{1}=2 a_{j}=2\left\{\sum_{i=0}^{j}\left(b_{i}-a_{i}\right)+a_{j}\right\}$.

Thus there exists the divided difference scheme which we denote by $\operatorname{DS}(\mathrm{a}, \mathrm{b}):=\mathrm{S}(\mathrm{a}, \mathrm{b})=2 \mathrm{~S}(\mathrm{c}, \mathrm{d})$, where the necessary condition (13) has been assumed. We then have:

Theorem 7. ( $\mathrm{C}^{1}$ convergence) If the divided difference scheme has discrete fundamental solutions which converge uniformly to a $\mathrm{C}^{0}$ limit, then the basic scheme $S(a, b)$ has discrete fundamental solutions which converge uniformly to a $\mathrm{C}^{1}$ limit $\varphi$. Moreover, the limit of the divided difference process (26) (i.e. with initial data $\left.\left\{d_{i}^{0}=\Delta \varphi_{i}^{O}, i \in z\right\}\right)$ is $\varphi^{\prime}$.

Theorem 7 suggests that the $\mathrm{C}^{0}$ convergence theory of sections 3 and 4 can be applied to the divided difference process in order to analyse differentiability. For $C^{0}$ convergence of this process, with respect to the diadic point parameterization, it is necessary that

$$
\sum_{j=0}^{m-1} a_{j}(1)=\sum_{j=0}^{m} b_{j}(1)=1 \text {. }
$$

This condition together with (11) is equivalent to:

Proposition 8. A necessary condition for uniform convergence of the divided difference process to a $\mathrm{C}^{0}$ limit with respect to the diadic point parameterization is that

$$
\sum_{j=0}^{m} a_{j}=\sum_{j=0}^{m} b_{j}=1 \text { and } \sum_{j=0}^{m}\left(b_{j}-a_{j}\right)=\frac{1}{2}
$$

It can be shown that if (29) holds, then the diadic point 
parameterization defined by (5) is an appropriate one for the analysis.If (29) does not hold, there may be some parameterization defined by differentpoints $\left\{t_{i}^{k}\right\}$ in which the limit curve may be differentiable. (The equivalent condition to (29) is then

$$
\sum_{j=0}^{m} a_{j}=\sum_{j=0}^{m} b_{j}=1 \text { and } \sum_{j=0}^{m} t_{i+j}^{k}\left(b_{j}-a_{j}\right)=t_{2 i+1}^{k+1}-t_{2 i}^{k+1},
$$

for parametric points $\left\{t_{i}^{k}\right\}$ which become dense in the limit.)

An immediate generalization of Theorem 7 is:

Theorem 9. (Cl convergence) Suppose there exist the v'th divided difference schemes $D^{v} S(a, b)=D S\left\{a\left({ }^{v-1}\right), b\left({ }^{v-1}\right)\right)=S\left(a\left({ }^{v}\right), b\left({ }^{v}\right)\right)$, $\mathrm{v}=0, \ldots, \ell$, where

$$
\sum a_{j}^{(v)}+\sum b_{j}^{(v)}=1, v=0, \ldots, \ell
$$

Then if $D^{\ell}-S(a, b)$ has discrete fundamental solutions which converge uniformly to a $\mathrm{C}^{0}$ limit, the basic scheme $\mathrm{S}(\mathrm{a}, \mathrm{b})$ has discrete fundamental solutions which converge uniformly to a $C^{l}$ limit $\varphi$.

Remark 10. Condition (30) implies that each v'th divided difference scheme has control point matrices with one eigenvalue unity. Since $D^{v} S(a, b)=2^{v} \Delta^{v} S(a, b)$, it can then be shown that the control matrices $\mathrm{C}^{0}$ and $\mathrm{C}_{1}$ of the difference scheme $\Delta \mathrm{S}(\mathrm{a}, \mathrm{b})$ (and hence of the basic scheme $\mathrm{S}(\mathrm{a}, \mathrm{b}))$ must have eigenvalues $1 / 2^{\mathrm{V}}, \mathrm{v}=1, \ldots, l$.

\section{A calculus of schemes}

\section{Given the basic scheme}

$$
S(a, b), \quad a=\left[a_{0}, \ldots, a_{m}\right], b=\left[b_{0}, \ldots, b_{m}\right]
$$

where $\Sigma \mathrm{a}_{i}=\Sigma \mathrm{b}_{i}=1$, we have defined the divided difference scheme $\operatorname{DS}(\mathrm{a}, \mathrm{b}):=\mathrm{S}(\mathrm{a}, \mathrm{b}), \mathrm{a}^{(1)}=\left[\mathrm{a}_{0}^{(1)}, \ldots \ldots, \mathrm{a}_{\mathrm{m}-1}^{(1)}\right], \mathrm{b}^{(1)}=\left[\mathrm{b}_{0}^{(1)}, \ldots \ldots, \mathrm{b}_{\mathrm{m}}^{(1)}\right]$, 
with coefficients given by (27), Conversely, there exists an integral scheme

$$
\begin{aligned}
\text { IS }(\mathrm{a}, \mathrm{b}):=\mathrm{S}\left(\mathrm{a}^{(-1)}, \mathrm{b}^{(-1)}\right), \mathrm{a}^{(-1)}=\left[\mathrm{a}_{0}^{(-1)}, \ldots \ldots, \mathrm{a}_{\mathrm{m}}^{(-1)}\right], \\
\mathrm{b}(-1)=\left[\mathrm{b}_{0}^{(-1)}, \ldots, \mathrm{b}_{\mathrm{m}+1}(-1)\right],
\end{aligned}
$$

whose divided difference scheme is the basic scheme, i.e. $\operatorname{DIS}(a, b)=S(a, b)$. The coefficients of this scheme are given by

$$
\left.\begin{array}{l}
a_{j}{ }^{(-1)}=\frac{1}{2}\left(a_{j}+b_{j}\right), j=0, \ldots, m \\
b_{0}{ }^{(-1)}=\frac{1}{2} b_{0}, b_{j}{ }^{(-1)}=\frac{1}{2}\left(a_{j-1}+b_{j}\right), j=1, \ldots, m+1
\end{array}\right\}
$$

Given that $S(a, b)$ has fundamental solution $\varphi$, let $D S(a, b)$ and IS $(a, b)$ have fundamental solutions $x$ and $\psi$ respectively. From Theorems 7 and 9 we have that if $x \in C^{0}(\infty, \infty)$, then $\varphi \varepsilon C^{1}(\infty, \infty)$ and $\psi \in C^{2}(-\infty, \infty)$ More precisely, we can relate the fundamental solutions in the following way:

Consider the divided difference scheme applied to the initial data $\Delta \varphi_{i}^{0}=\Delta \delta i, 0 / i \in z$ Then the limit curve is

$$
\varphi^{\prime}(t) \equiv X(t+1>-X(t)
$$

Thus, noting the local support of $X$,

$$
\varphi(t)=\int_{t}^{t+1} x(s) d s=\int_{-\infty}^{\infty} x(t-s) B l(s) d s=x * B 1(t),
$$

where

$$
\operatorname{B1}(s)=\left\{\begin{array}{l}
1, \leq s \leq 0 \\
0, \text { otherwise }
\end{array}\right.
$$

Applying this convolution result to the integral scheme thus gives: 
Theorem 11. Let $S(a, b)$ have discrete fundamental solutions which converge uniformly to $\varphi \in C(-\infty, \infty)$. Then IS $(a, b)$ has discrete fundamental solutions which converge uniformly to

$$
\psi=\varphi \star B_{1}
$$

and, in general, $I^{\ell} \mathrm{S}(\mathrm{a}, \mathrm{b}), \ell \geq 1$, has discrete fundamental solutions which converge uniformly to

$$
\psi \star B_{\ell}:=\psi \star B_{1} \star \cdot . \cdot \star B_{1} .
$$

We thus see that $\ell^{\prime}$ th order integral schemes (and $C^{\ell}$ basic schemes) have fundamental solutions which are defined by convolutions with $\ell^{\prime}$ th order B-splines, which confirms a conjecture of C.A. Micchelli.

\section{An interpolatory subdivision scheme for surfaces}

We have so far described a theory for the analysis of convergence of univariate uniform subdivision algorithms defined by a rule of the form (1). The motivation for this work is the specific example of the 4-point interpolatory curve scheme defined by (2). Application of the theory to this specific case (with $L=2$ in the matrix analysis of section 4) gives $-0.375<\mathrm{w}<0.39$ and $0<\mathrm{w}<0.154$ as sufficient conditions for $a C^{\circ}$ and $C^{1}$ limit curve respectively, see Dyn et al (1988). Taking higher values of $L$ and using similarity transformations on the control point matrices suggested by M.J.D. Powell gives improved ranges for w. For example numerical experiments indicate that $|\mathrm{w}|<1 / 2$ is a sufficient condition for a $\mathrm{C}^{0}$ limit, where for negative $\mathrm{w}$ we have used the result of Micchelli and Prautzsch (1987b) using the positivity of the coefficients in (2) $(|w|) l<1 / 2$ is also necessary by application of Corollary 5).

We conclude by describing a bivariate interpolatory subdivision scheme for surfaces whose parameterization can be defined on a "type 1" regular triangulation. Clearly, tensor product type surface 
schemes can be derived immediately from the univariate theory but our interest here is in the development of triangular based schemes. The scheme is defined as follows:

Let $\mathrm{f}^{\mathrm{k}}$ denote a control polygon in $\mathrm{R}^{3}$ with control points $f_{i}^{k}, j \in R^{3}, \quad(i, j) \in z^{2}$, and consisting of triangular faces with vertices $\left\{f_{i, j}, f_{i+1, j}, f_{i, j+1}\right)$ and $\left\{f_{i+1, j}, f_{i+1,1} f i, j+1\right\} . T h e n f^{k+1}$ has vertices defined by the rule

$$
\begin{aligned}
& \mathrm{f}_{2 i, 2 j}^{\mathrm{k}+1}=\mathrm{f}_{i, j}^{\mathrm{k}} \\
& f^{k+1} 2 i+1,2 j=\frac{1}{2}\left[f_{i, j}^{k}+f_{i+1, j}^{k}\right]+2 \omega\left[f_{i, j+1}^{k}+f_{i+1, j-1}^{k}\right] \\
& -\omega\left[f_{i-1, j+1}^{k}+f_{i+1, j+1}^{k}+f_{i, j-1+f_{i}^{k}+2, j-1}^{k}\right] \\
& \begin{aligned}
f_{2 i, 2 j}^{k+1}+1 & =\frac{1}{2}\left[f_{i, j}^{k}+f_{i, j+1}^{k}\right]+2 \omega\left[f_{i+1, j}^{k}+f_{i}^{k}\right. \\
& -\omega\left[f_{i+1, j-1}^{k}+f_{i+1, j}^{k}+f_{i+1, j+2}^{k}\right]
\end{aligned} \\
& f_{2 i+1,2 j+1}^{k}=\frac{1}{2}\left[f_{i+1, j}^{k}+f_{i, j+1}^{k}\right]+2 \omega\left[f_{i+1, j+1}^{k}\right] \\
& -\omega\left[f_{i-1, j+1}^{k}+f_{i+1, j-1}^{k}+f_{i, j+2}^{k}+f_{i+2, j}^{k}\right]
\end{aligned}
$$

As with the univariate rule (2), varying w in (40) gives some control on the shape of the limit surface. The case $\mathrm{w}=0$ gives $\mathrm{f}^{\mathrm{k}+1}=\mathrm{fk}$ for all $k$ and hence the limit surface is the initial control polygon $\mathrm{f}^{0}$. The case $\mathrm{w}=1 / 16$ corresponds to a rule which has bivariate cubic polynomial precision with respect to the diadic point parameterization: $f_{i, j}^{k}$ being defined at

$$
t_{i, j}^{k}:=\left(i / 2^{k}, j / 2^{k}\right.
$$

The scheme (40) then corresponds to a symmetric rule defined on a 
uniform subdivision of a "type 1" regular triangulation.

A convergence analysis of this algorithm is currently being developed which suggests that the limit surface will be $\mathrm{C}^{1}$ for a range of $\mathrm{w}$ which includes $\mathrm{w}=1 / 16$. This indicates the existence of a $\mathrm{C}^{1}$ interpolant on a regular triangulation whose fundamental solutions (i.e. cardinal basis functions) have local support. The subdivision algorithm is illustrated by the following example:

The first figure of Figures 2 shows a set of control points defined on the surface of a sphere with two control points pulled away from the spherical surface to give the initial control polygon $f^{0}$. The remaining Figures show a shaded picture description of the results of the subdivision algorithm through four levels of recursion with $w=1 / 16$ and where appropriate additional control points have been defined as boundary conditions on the algorithm external to the surface shown. The results indicate a smoothing process suggested by a $C^{1}$ limit.

\section{Acknowledgements}

This work was supported by the U.K. Science and Engineering Research Council grants GR/E/25139 and 26594; the U.S.A.-Israel Binational Science Foundation grant 86-00243; and the Israel Academy of Science and Humanities grant 548-86. We are also pleased to acknowledge A.Betashvilli in Israel and R.Qu in the U.K.for their contribution to this work.

\section{References}

Dyn, N.,Gregory, J.A., and Levin, D. (1987), A 4-point interpolatory subdivision scheme for curve design, Computer Aided Geometric Design 4, pp 257-268.

Dyn, N., Gregory, J.A. and Levin, D. (1988), Analysis of uniform binary subdivision schemes for curve design, Brunei University preprint. 
Micchelli, C.A. and Prautzsch, H. (1987a), Uniform refinement of curves, IBM Research Centre preprint.

Micchelli, C.A. and Prautzsch, H. (1987b), Refinement and subdivision for spaces of integer translates of a compactly supported function, proceedings of the Dundee Numerical Analysis Conference.

Prautzsch, H. and Micchelli, C.A. (1987C), Computing curves invariant under halving. Computer Aided Geometric Design 4, pp 133-140.
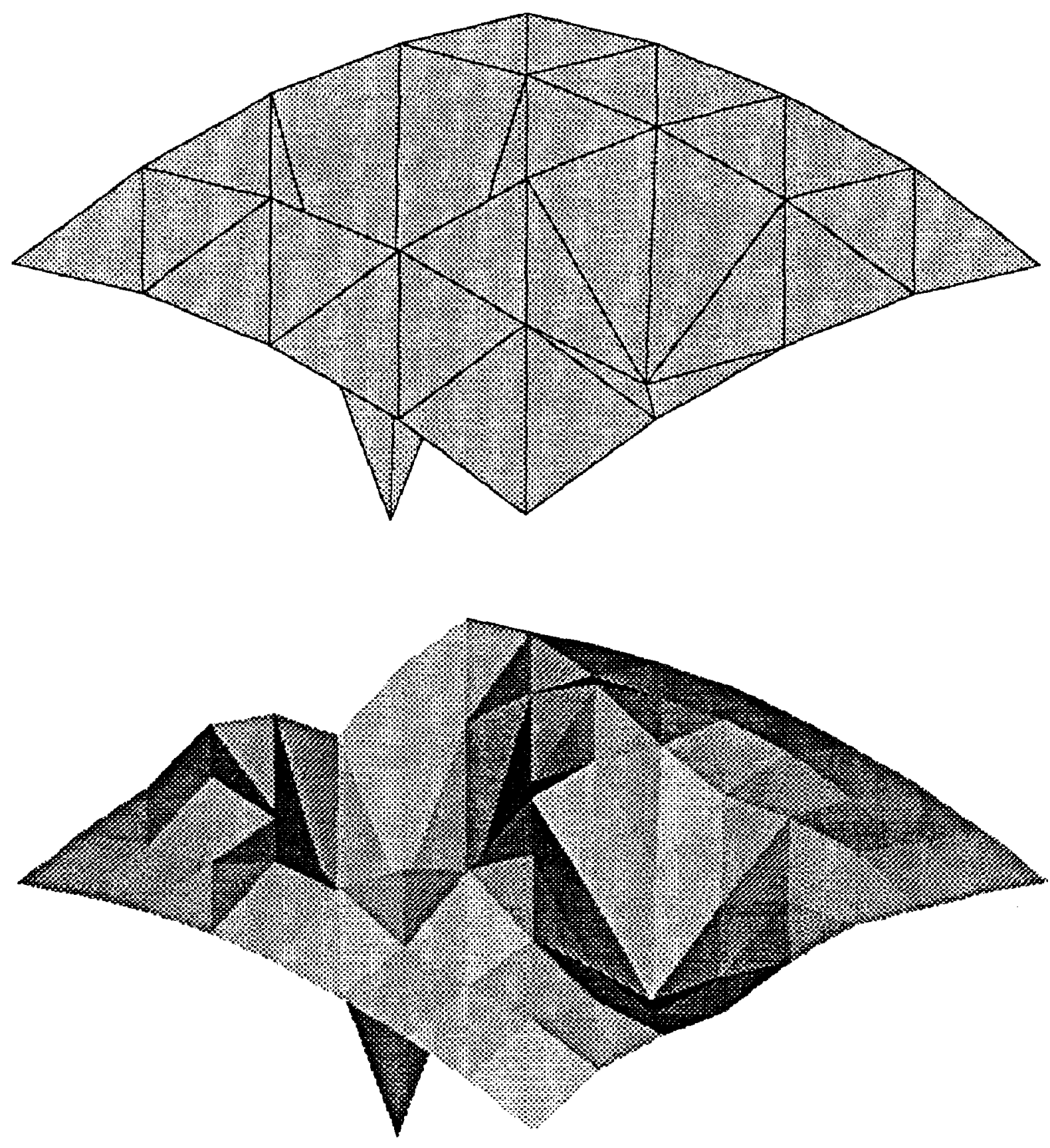

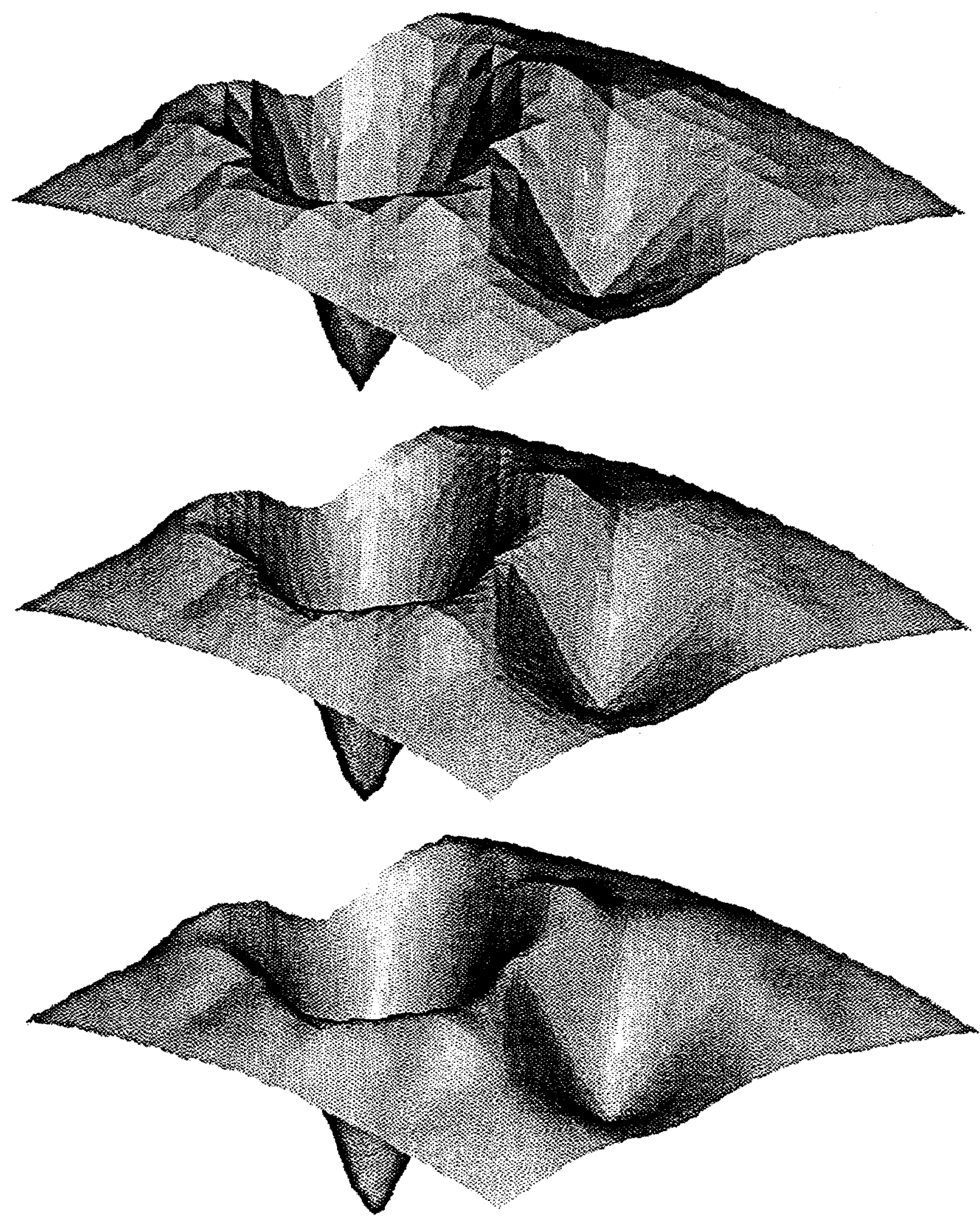

Figures 2. Example of interpolatory surface algorithm

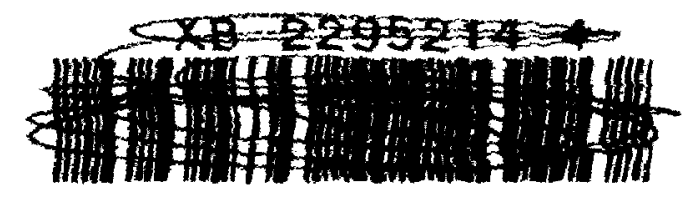

